Nephrologe 2021 · 16:66-70

https://doi.org/10.1007/s11560-020-00473-z

Angenommen: 20. November 2020

Online publiziert: 14 . Dezember 2020

(c) Springer Medizin Verlag GmbH, ein Teil von

Springer Nature 2020

Redaktion

T. Feldkamp, Kiel

O. Witzke, Essen

Die Erkrankung COVID-19 (,coronavirus disease $2019^{\prime \prime}$ ) betrifft neben der Lunge als Ort der Hauptmanifestation auch weitere Organe, insbesondere die Nieren. Im Rahmen schwerer Verläufe und im zeitlichen Zusammenhang mit der progredienten respiratorischen Verschlechterung entwickelt sich häufig ein akutes Nierenversagen, welches mit einer schlechten Prognose assoziiert ist. Das Spektrum der Nierenbeteiligung bei COVID19 reicht von subklinischen Urinauffälligkeiten bis zum schweren dialysepflichtigen Nierenversagen.

\section{Prognostisch bedeutsam: frühe Nierenbeteiligung bei SARS- CoV-2}

Mehrere Studien konnten zeigen, dass viele der zuvor nierengesunden Patienten bereits zu Beginn einer COVID-19Erkrankung eine Nierenbeteiligung mit Nachweis einer Albuminurie (und/oder Hämaturie) aufweisen [1, 2]. Diese frühen Zeichen einer Nierenbeteiligung sind für den weiteren Verlauf einer COVID19-Erkrankung prognostisch bedeutsam [3]: So verschlechtert eine Nierenbeteiligung bei COVID-19 das Patienten-Outcome dramatisch und erhöht die Mortalität um das bis zu 10-Fache $[1,4]$. Das akute Nierenversagen betrifft hauptsäch-

Teile dieses Beitrags basieren auf den Publikationen Rohn et al. (2020), Der Nephrologe 15:210-215, https://doi.org/10.1007/s11560020-00444-4, und Rohn et al. (2020), Der Gastroenterologe 15:471-476, https://doi.org/10. 1007/s11377-020-00484-1.

\author{
A. Gäckler ${ }^{1} \cdot$ H. Rohn ${ }^{2} \cdot$ O. Witzke ${ }^{2}$ \\ ${ }^{1}$ Klink für Nephrologie, Universitätsmedizin Essen, Universität Duisburg-Essen, Essen, Deutschland \\ ${ }^{2}$ Klinik für Infektiologie, Westdeutsches Zentrum für Infektiologie, Universitätsmedizin Essen, Universität \\ Duisburg-Essen, Essen, Deutschland
}

\title{
Akutes Nierenversagen bei COVID-19
}

lich Patienten mit respiratorischer Insuffizienz, insbesondere solche mit der Notwendigkeit einer mechanischen Beatmung, und steht häufig auch in zeitlichem Zusammenhang mit einer erforderlichen Intubation [5].

\section{Akute Nierenschädigung - eine Folge der SARS-CoV-2- Infektion?}

Das Auftreten eines akuten Nierenversagens ist unabhängig von seiner Genese mit einer verschlechterten Erkrankungsprognose assoziiert [6] und ist auch im Rahmen von COVID-19 ein unabhängiger Prädiktor für die Mortalität [5, 6]. Grundsätzlich werden hier ein negativer Einfluss des gestörten Wasser- und Säure-Basen-Haushalts, eine Unterdrückung der Immunantwort sowie eine fehlregulierte Inflammation vermutet. Ein akutes Nierenversagen trägt häufig zu einer schlechteren Steuerbarkeit des Volumenhaushalts bei, und eine (pulmonale) Überwässerung kann den Gasaustausch und auch die Lungenschädigung im Rahmen einer bereits bestehenden respiratorischen Insuffizienz weiter verstärken [7].

\section{》) Das akute Nierenversagen ist bei COVID-19-Patienten häufig anzutreffen}

Das akute Nierenversagen ist bei COVID19-Patienten häufig anzutreffen: In großen Beobachtungsstudien entwickelten 5-50\% der hospitalisierten Patienten mit COVID-19 ein akutes Nierenversagen unterschiedlichen Schweregrads [5, 8], in einer französischen Studie lag die Inzidenz sogar bei $80 \%$ [9]. In großen Kohortenstudien aus den USA lag der Anteil der Patienten mit akutem dialysepflichtigen Nierenversagen bei 5-20\% [1]. Unter den intensivpflichtigen Patienten entwickelten sogar fast zwei Drittel ein akutes Nierenversagen, von denen 25-45\% eine Dialysetherapie benötigten [5]. Diese Daten wurden durch Registerstudien aus anderen Ländern bestätigt. Eine Auswertung der Daten von über 10.000 AOK-versicherten Patienten aus mehr als 900 deutschen Krankenhäusern zeigte, dass allein $27 \%$ der beatmungspflichtigen COVID-19-Patienten während des Krankenhausaufenthalts ein dialysepflichtiges akutes Nierenversagen entwickelten [10]. Risikofaktoren für die Entwicklung eines akuten Nierenversagens im Rahmen von COVID-19 finden sich in der $\bullet$ Infobox 1.

Infobox 1 Demographische Risikofaktoren für die Entwicklung eines akuten Nierenversagens im Rahmen von COVID-19 (nach [5, 11])

- höheres Alter

- Übergewicht

- Diabetes mellitus

- Herz-Kreislauf-Erkrankungen

- chronische Nierenkrankheit

- schwarze Hautfarbe

- genetische Risikofaktoren (APOL1[Apolipoprotein L1]-Genotyp, ACE2 [,angiotensinconverting enzyme 2"]-Polymorphismus)

- Immunsuppression

- Raucheranamnese

- Hypertonie 
Hier steht eine Anzeige.

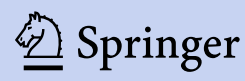


Ob die hohe Inzidenz an akutem Nierenversagen bei COVID-19 die Schwere der systemischen Erkrankung wiederspiegelt oder ob ein akutes Nierenversagen auch eine separate (von der Erkrankungsschwere) unabhängige Organmanifestation der SARS-CoV-2(„,severe acute respiratory syndrome coronavirus 2“)-Infektion darstellen kann, ist Gegenstand aktueller intensiver Forschung.

\section{Mögliche pathophysiologische Mechanismen}

Neben einem direktem Virusbefall der Niere sind vielfältige weitere Mechanismen denkbar, die zu einer insgesamt multifaktoriellen Genese des akuten Nierenversagens beitragen können [12]. So kann es im Rahmen der respiratorischen Insuffizienz zu einer Hypooxygenierung und Rechtsherzbelastung mit konsekutiver Entstehung eines ischämisch bedingten akuten Nierenversagens kommen. Sehr wahrscheinlich virusassoziierte Zytokinfreisetzung und Komplementaktivierung können ebenfalls zu Inflammation und Zellschädigung beitragen [13]. Zugleich wäre eine Verstärkung des Alveolarschadens durch die Komplementaktivierung denkbar. Auch die damit einhergehende Hyperkoagulabilität könnte durch die Entstehung von Mikrothromben in Lunge und Niere direkt und indirekt über eine weitere Verminderung der Oxygenierung bei erhöhtem pulmonalen Widerstand zu einer Nierenschädigung beitragen. Zusätzliche weitere mögliche Faktoren, welche die Nierenschädigung triggern können, sind die Toxizität von Arzneimitteln und die häufig im Rahmen der Erkrankung beobachtete Rhabdomyolyse [4, 14].

Spezifische histopathologische renale Veränderungen, wie sie beispielsweise bei Hantavirusinfektionen oder Polyomavirusnephropathie beschrieben werden, konnten bei COVID-19-Patienten mit akutem Nierenversagen bislang nicht identifiziert werden [15]. Der mit Abstand häufigste histopathologische Befund in den Nieren bei COVID-19Patienten ist eine akute Tubulusnekrose im Bereich des proximalen Tubulus. Auch das durch Urinanalyse erhobe-

Nephrologe 2021 · 16:66-70 https://doi.org/10.1007/s11560-020-00473-z

(c) Springer Medizin Verlag GmbH, ein Teil von Springer Nature 2020

\section{A. Gäckler · H. Rohn · O. Witzke}

\section{Akutes Nierenversagen bei COVID-19}

\section{Zusammenfassung}

Das akute Nierenversagen („,acute kidney injury", AKI) ist eine häufige Komplikation bei COVID-19 ("coronavirus disease 2019“). Es geht häufig mit der progredienten respiratorischen Verschlechterung einher und ist mit einer erhöhten Morbidität und Mortalität assoziiert. Das AKI ist vermutlich multifaktorieller Genese, wobei auch ein direkter Virusbefall der Niere eine Rolle zu spielen scheint. Spezifische Behandlungsverfahren für das COVID-19-assoziierte Nierenversagen existieren aktuell nicht. Auch die Rolle extrakorporaler Verfahren in der COVID-19-
Behandlung konnte bislang nicht geklärt werden. Aktuelle Daten deuten auf eine anhaltende Nierenfunktionseinschränkung nach COVID-19-assoziiertem AKI hin, sodass eine Reevaluation der Nierenfunktion nach stattgehabter Erkrankung empfohlen werden sollte.

\section{Schlüsselwörter}

Coronavirus · Komplikationen · Pathophysiologie · Extrakorporale Blutreinigung · Nierenfunktionseinschränkung

\section{Acute renal failure in COVID-19}

Abstract

Acute kidney injury (AKI) is a frequent complication in coronavirus disease 2019 (COVID-19). It is often linked to progressive respiratory failure and is associated with increased morbidity and mortality. The AKI is presumably of multifactorial origin, whereby direct viral infestation of the kidneys also seems to be involved. Specific treatment procedures for AKI associated with COVID-19 are currently missing. In addition, the role of extracorporeal procedures in the treatment of COVID-19 could so far not be clarified. Latest data indicate persistent loss of renal function following COVID-19-associated AKI. Therefore, a re-evaluation of renal function following recovery from COVID-19 should be recommended.

Keywords

Coronavirus - Complications - Pathophysiology · Extracorporeal blood purification · Renal insufficiency ne Schädigungsmuster entspricht einer (vorwiegend) akuten tubulären Schädigung, einer interstitiellen Nephritis oder einer Kombination von beidem [9]. Zudem konnten in einigen Fällen Zeichen einer thrombotischen Mikroangiopathie gefunden werden [16]. Diese Befunde sind gut vereinbar mit der Hypothese einer multifaktoriellen Genese des akuten Nierenversagens bei SARS-CoV-2.

\section{I) Der hohe Nierentropismus von SARS-CoV-2 kann z.T. durch dessen Affinität für das ACE2 erklärt werden}

Eine derzeit stark diskutierte Frage ist, inwieweit eine direkte Virusaffektion der Niere zur Pathogenese beiträgt. So konnten in einzelnen Obduktionsstudi- en SARS-CoV-2-Antigene in menschlichen Nieren nachweisen werden [17-19]. Auch eine häufig nicht vorhandene Differenzierung von Patienten mit und ohne akutes Nierenversagen anhand inflammatorischer Parameter (C-reaktives Protein [CRP] und Ferritin) könnte auf einen virusspezifischen Schaden hindeuten [9].

Der hohe Nierentropismus von SARSCoV-2 kann z. T. durch die Affinität des Virus für das membrangebundene ACE2 erklärt werden [20]. SARS-CoV-2 nutzt ACE2 als „Türöffner“, um Zugang zu seinen Zielzellen zu erhalten [21, 22]. ACE2 findet sich v. a. in Alveolarzellen der Lunge, aber auch in anderen Organen, darunter der Niere, wo es insbesondere von proximalen Tubuluszellen und glomerulären Parietalzellen, zum Teil aber auch von Podozyten exprimiert wird [23]. Der Virusbefall der Niere scheint auch funk- 

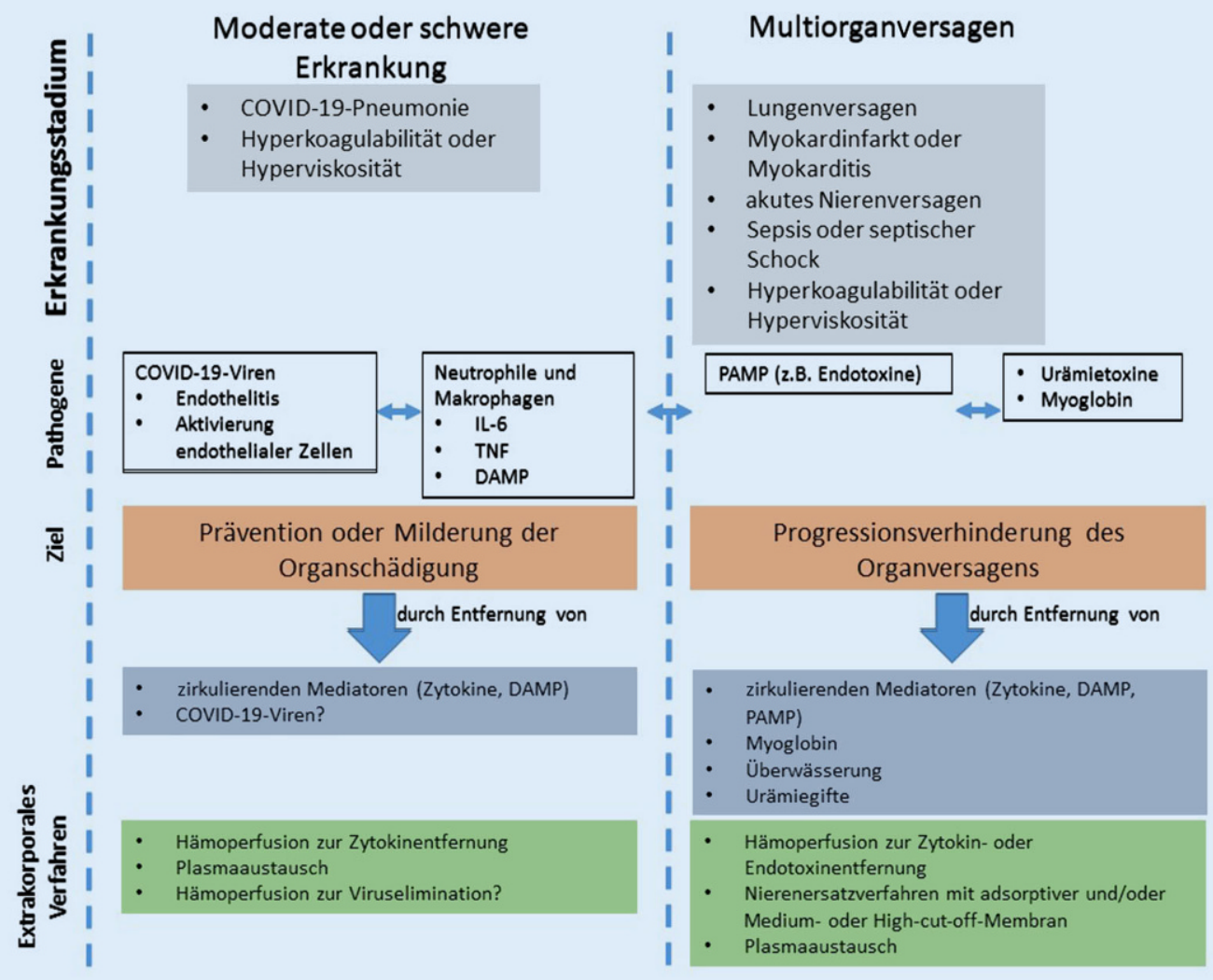

Abb. $1 \varangle$ Mögliche extrakorporale Verfahren als Behandlungsoption bei COVID-19 ("coronavirus disease 2019"). DAMP "damage-associated molecular patterns", PAMP "pathogen-associated molecular patterns", TNFTumornekrosefaktor, IL Interleukin. (Modifiziert nach [11])

tionell relevant zu sein, da eine Assoziation mit dem Auftreten eines akuten Nierenversagens beobachtet werden konnte [24].

\section{ACE-Hemmer und SARS-CoV-2}

Aus den bisherigen epidemiologischen Daten geht hervor, dass über zwei Drittel der Patienten, die an COVID-19 starben, an Diabetes oder Herz-Kreislauf-Erkrankungen litten [25]. Die meisten dieser Patienten erhielten eine leitliniengerechte blutdrucksenkende Therapie mit ACEHemmern oder Angiotensinrezeptorblockern (ARB). Diese beiden Medikamentengruppen sind in der Lage, die ACE2Expression in der Niere und im Herzen um das 2- bis 5-Fache zu erhöhen [26, 27].

Nach dem derzeitigen Wissensstand gibt es jedoch keine Belege für einen Zusammenhang zwischen der Einnahme von ACE-Hemmern bzw. ARB und einem schweren COVID-19-Verlauf [28, 29]. In ihren offiziellen Stellungnahmen empfehlen die European Society of Hy- pertension (ESH) und die European Society of Cardiology (ESC) daher, dass die Blutdruckmedikamente bei Patienten mit einem Risiko für COVID-19 oder bei Patienten, bei denen bereits COVID-19 diagnostiziert wurde, nach Möglichkeit nicht verändert werden sollten.

\section{Einsatz extrakorporaler Verfahren bei COVID-19}

Inflammatorische Zytokine, DAMP („damage-associated molecular patterns“) und PAMP („pathogen-associated molecular patterns") einschließlich Endotoxinen und COVID-19-Viruspartikeln stehen in Verdacht, die Entwicklung des Multiorganversagens und die Mortalität kritisch kranker Patienten mit COVID-19 mitzubestimmen. Extrakorporale Verfahren können zur Entfernung dieser Substanzen und damit potenziell zur Behandlung kritisch kranker Patienten mit Organversagen eingesetzt werden (•Abb. 1). Aufgrund der eingeschränkten Datenlage besteht zum jetzigen Zeitpunkt jedoch kein Konsens über Grenzwerte spezifischer laborchemischer oder klinischer Kriterien in Bezug auf den Einsatz, das Monitoring oder den Abbruch solcher Verfahren [11]. Hier sind weitere Studien dringend erforderlich.

\section{Entwicklung der Nierenfunktion nach akutem Nierenversagen}

Aufgrund der geringen Zeitspanne seit Beginn der COVID-19-Pandemie liegen bislang nur eingeschränkte Daten im Hinblick auf die langfristige Prognose der Nierenfunktion nach akutem Nierenversagen im Rahmen von COVID19 vor. Während in einer Studie eine Erholung der Nierenfunktion beschrieben wurde [4], weist die Studie von Gupta et al. auf eine deutlich über den Intensivaufenthalt hinausreichende persistierende Dialysepflichtigkeit hin [30]. So verblieb ein Sechstel der Patienten, die im Rahmen von COVID-19 intensivmedizinisch betreut worden waren und eine Nierenersatztherapie erhalten 
hatten, auch 60 Tage nach Entlassung von der Intensivstation dialysepflichtig.

Ob Patienten - ähnlich der beschriebenen pulmonalen Fibrose nach COVID-19 - auch eine renale Fibrose mit Progression zur chronischen Nierenerkrankung erleiden können, ist bislang unklar. Bei Patienten mit COVID-19-assoziiertem akuten Nierenversagen sollte daher 2 bis 3 Monate nach Entlassung aus der stationären Betreuung eine erneute Beurteilung der Nierenfunktion erfolgen [11].

\section{Fazit für die Praxis}

\section{- Das akute Nierenversagen ist eine häufige Organmanifestation bei Patienten mit COVID-19 („,coronavirus disease 2019"). \\ - Das akute Nierenversagen führt zu einer deutlichen Verschlechterung der erhöht die Mortalität. \\ - Die multifaktorielle Genese des akuten Nierenversagens erfordert eine interdisziplinäre Betreuung. \\ - Zudem sollte im Hinblick auf die lang- fristige Prognose genesener COVID- 19-Patienten eine nephrologische Nachsorge erfolgen.}

\section{Korrespondenzadresse}

PD Dr. med. A. Gäckler

Klink für Nephrologie, Universitätsmedizin Essen, Universität Duisburg-Essen Hufelandstr. 55, 45147 Essen, Deutschland anja.gaeckler@uk-essen.de

\section{Einhaltung ethischer Richtlinien}

Interessenkonflikt. A. Gäckler erhielt Referentenhonorare von Ablynx/Sanofi, Alexion und Novartis. 0 . Witzke erhielt Forschungsstipendien für klinische Studien, Referentenhonorare und Reisekosten von Amgen, Alexion, Astellas, Basilea, Biotest, BristolMyers Squibb, Correvio, Chiesi, Gilead, Hexal, Janssen, Dr. F. Köhler Chemie, MSD, Novartis, Roche, Pfizer, Sanofi, TEVA and UCB. H. Rohn gibt an, dass kein Interessenkonflikt besteht.

Für diesen Beitrag wurden von den Autoren keine Studien an Menschen oder Tieren durchgeführt. Für die aufgeführten Studien gelten die jeweils dort angegebenen ethischen Richtlinien.

\section{Literatur}

1. Cheng Y, Luo R, Wang K, Zhang M, Wang Z, Dong L et al (2020) Kidney disease is associated with inhospital death of patients with COVID-19. Kidney Int 97(5):829-838

2. Cheng Y, Luo R, Wang X, Wang K, Zhang N, Zhang $M$ et al (2020) The incidence, risk factors, and prognosis of acute kidney injury in adult patients with coronavirus disease 2019. Clin J Am Soc Nephrol 15(10):1394-1402

3. Gross O, Moerer O, Weber M, Huber T, Scheithauer S (2020) COVID-19-associated nephritis: early warning for disease severity and complications? Lancet 395(e10236):e87-e88

4. Pei G, Zhang Z, Peng J, Liu L, Zhang C, Yu C et al (2020) Renal involvement and early prognosis in patients with COVID-19 pneumonia. J Am Soc Nephrol 31(6):1157-1165

5. Hirsch J, Ng J, Ross D, Sharma P, Shah H, Barnett $R$ et al (2020) Acute kidney injury in patients hospitalized with COVID-19. Kidney Int 98(1):209-218

6. Kellum J, van Till J, Mulligan G (2020) Targeting acute kidney injury in COVID-19. Nephrol Dial Transplant 35(10):1652-1662

7. Bellomo R, Kellum J, Ronco C, Wald R, Martensson J, Maiden Metal (2017) Acute kidney injury in sepsis. Intensive Care Med 43(6):816-828

8. Chan L, Chaudhary K, Saha A, Chauhan K, Vaid A, Baweja M et al (2020) Acute kidney injury in hospitalized patients with Covid-19. medRxiv. https://doi.org/10.1101/2020.05.04.20090944

9. Rubin S, Orieux A, Prevel R, Garric A, Bats M, Dabernat $S$ et al (2020) Characterization of acute kidney injury in critically ill patients with severe coronavirus disease 2019. Clin Kidney J 13(3):354-361

10. KaragiannidisC,MostertC, HentschkerC,VoshaarT, Malzahn J, Schillinger G et al (2020) Case characteristics, resource use, and outcomes of 10021 patients with COVID-19 admitted to 920 German hospitals: an observational study. Lancet RespirMed 8(9):853-862

11. Nadim M, Forni L, Mehta R, Connor M Jr, Liu K, Ostermann $\mathrm{M}$ et al (2020) COVID-19-associated acute kidney injury: consensus report of the 25th Acute Disease Quality Initiative (ADQI) workgroup. Nat Rev Nephrol. https://doi.org/10.1038/s41581 020-00356-5

12. Ronco C, Reis T, Husain-Syed F (2020) Management of acute kidney injury in patients with COVID-19. Lancet Respir Med 8:738-742

13. Risitano A, Mastellos D, Huber-Lang M, Yancopoulou D, Garlanda C, Ciceri F et al (2020) Complement as a target in COVID-19? Nat Rev Immunol 20(6):343-344

14. Mohamed M, Lukitsch I, Torres-Ortiz AE, Walker J, Varghese V, Hernandez-Arroyo C et al (2020) Acute kidney injury associated with coronavirus disease 2019 in urban New Orleans. Kidney 1(7):614-622

15. Remmelink M, De Mendonca R, D'Haene N, De Clercq S, Verocq C, Lebrun L et al (2020) Unspecific post-mortem findings despite multiorgan viral spread in COVID-19 patients. Crit Care 24(1):495

16. Menter T, Haslbauer J, Nienhold R, Savic S, Hopfer H, Deigendesch N et al (2020) Postmortem examination of COVID-19 patients reveals diffuse alveolar damage with severe capillary congestion and veriegated findings in lungs and other organs suggesting vascular dysfunction. Histopathology 77(2):198-209

17. Chen T, Wu D, Chen H, Yan W, Yang D, Chen G et al (2020) Clinical characteristics of 113 deceased patients with coronarvirus disease 2019 : retrospective study. BMJ 368:m1295

18. Martinez-Rojas M, Vega-Vega O, Bobadilla N (2020) Is the kidney a target of SARS-CoV-2? Am J Physiol Renal Physiol 318(6):F1454-F1462

19. Puelles V, Lütgehetmann $M$, Lindenmeyer $M$, Sperhake J, Wong M, Allweiss L et al (2020) Multiorgan and renal tropism of SARS-CoV-2. NEngl JMed 383(6):590-592

20. Wan Y, Shang J, Graham R, Baric R, Li F (2020) Receptor recognition by the novel coronavirus from Wuhan: an analysis based on decade-long structural studies of SARS coronavirus. J Virol 94(7):e127

21. Hoffmann M, Kleine-Weber $H$, Schroeder $S$, Krüger N, Herrler T, Erichsen S et al (2020) SARSCoV-2 cell entry depends on ACE2 and TMPRSS2 and is blocked by clinically proven protease inhibitor. Cell 181(2):271-280

22. Yan R, Zhang Y, Li Y, Xla L, Guo Y, Zhou Q (2020) Structural basis for the recognition of SARS-CoV-2 by full-length human ACE2. Science 367(6485):1444-1448

23. Su H, Yang M, Wan C, Yi L, Tang F (2020) HY Z, et al. Renal histopathological analysis of 26 postmortem findings of patients with COVID-19 in China. Kidney Int 98(1):219-227

24. Braun $F$, Lütgehetmann $M$, Pfefferle $S$, Wong $M$, Carsten A, Lindenmeyer M et al (2020) SARS-CoV-2 renal tropism associates with acute kidney inury. Lancet 396(10251):597-598

25. Remuzzi A, Remuzzi G (2020) COVID-19 and Italy: what next? Lancet 395(10231):1225-1228

26. Ishiyama Y, Gallagher P, Averill D, Trallant E, Brosnihan K, Ferrario C (2004) Upregulation of angiotensin-converting enzyme 2 after myocardial infarction by blockade of angiotensin II receptors. Hypertension 43(5):970-976

27. Wysocki J, Lores E, Ye M, Soler M, Batlle D (2020) Kidney and lung ACE2 expression after an ACE inhibitor or an ANG II receptor blocker: implications for COVID-19. J Am Soc Nephrol 31(9):1941-1943

28. Danser A, Epstein M, Batlle D (2020) Reninangiotensin system blockers and the COVID19 pandemic: at present there is no evidence to abandon renin-angiotensin system blockers. Hypertension 75(6):1382-1385

29. Sparks M, South A, Welling P, Luther J, Cohen J, Byrd J et al (2020) Sound scinece before quick judgement regarding RAS blockade in COVID-19. Clin J Am Soc Nephrol 15(5):714-716

30. Gupta A, Coca S, Chan L, Melamed M, Brenner S, Hayek $S$ et al (2020) AKI treated with renal replacement therapy in critically ill patients with COVID-19. J Am Soc Nephrol. https://doi.org/10. 1681/ASN.2020060897 